\title{
Study on Bayes Semiparametric Regression
}

\author{
Abdulhussein Saber AL-Mouel and Ameera Jaber Mohaisen \\ Mathematics Department College of Education for Pure Sciences, AL-Basrah University, Iraq \\ Email: ameera.jaber@yahoo.com
}

\begin{abstract}
In this paper, Bayesian approach based on Markov chain Monte Carlo (MCMC) to (fully) Semiparametric regression problems is described as a mixed model using a convenient connection between penalized splines and mixed models. We investigate the inferences on the model coefficients under some conditions on the prior, as well as studying some properties of the posterior distribution and identifying the analytic form of the Bayes factors.
\end{abstract}

Keywords: Semiparametric regression, penalized spline, mixed model, bayes approach, prior distribution, posterior distribution, bayes factor.

\section{Introduction}

Semiparametric regression models have been investigated by many researchers. Lenk (1999) presented the Bayesian inference of a semiparametric regression model using a Fourier representation[6]. Natio (2002) studied semiparametric regression with multiplicative adjustment[10]. Also Brezger et al. (2002) investigated and analyzed Bayesian semiparametric models[1]. Ruppert et al. (2003) introduced semiparametric regression models based on penalized regression splines and mixed models[12]. Tsiatis and MA (2004) studied locally efficient semiparametric estimators for functional measurements error models by defining after projecting the score vector with respect to the parameter $\beta$ on to the nuisance tangent space for the nonparametric conditional distribution of $X$ given $Z$, where $Z$ is the predictor variable measured precisely[14]. In (2007), Yuan and DE Gooijer presented semiparametric regression with kernel error model[16]. Also in (2007), Jensen and Maheu studied bayesian semiparametric stochastic volatility modeling[3]. Choi, Lee and Roy (2008) investigated the large sample property of the Bayes factor for testing the parametric null model against the Semiparametric alternative model[2].

Wand (2009) presented semiparametric regression and graphical models[15]. Tarmaratram (2011) proposed a robust estimation method in semiparametric regression models for penalized regression splines that can be used in the presence of outliers in the response variable, and studied a robust version of the model selection criterion AIC, Akaiki's information criterion for regression models where S- and MM- estimators are used for estimation[13]. Pelenis (2012) studied Bayesian semiparametric regression that considered a Bayesian estimation of restricted conditional moment model with linear regression as a particular example[11]. Mohaisen and Abdulhussain investigated Bayesian semiparametric regression based on penalized spline[7-9].

This paper came to shed light on the semiparametric regression model which has two parts, the parametric (first part) is assumed to be linear function of p-dimensional covariates and the nonparametric (second part) is assumed to be a smooth penalized spline, as well as the error term which has normal distribution with mean zero and variance $\sigma_{\varepsilon}^{2}$. We can represent semiparametric regression model as a mixed model by using a convenient connection between penalized splines and mixed model.

In this paper, Bayesian approach based on Markov chain Monte Carlo (MCMC) to (fully) Semiparametric regression problems is described as a mixed model using a convenient connection between penalized splines and mixed models. We investigate the inferences on the model coefficients under some conditions on the prior, as well as studying some properties of the posterior distribution and identifying the analytic form of the Bayes factors. 


\section{Description of the Problem and the Prior Distribution}

Consider the model:

$$
y_{i}=\sum_{j=0}^{p} \beta_{j} x_{j i}+g\left(x_{p+1, i}\right)+\varepsilon_{i}, i=1,2, \ldots, n
$$

where $\sum_{j=1}^{p} \beta_{j} x_{j i}$ is the parametric part which assumed to be linear function of p-dimensional covariates, $g\left(x_{p+1, i}\right)$ the nonparametric part and the unobserved errors $\varepsilon_{1}, \varepsilon_{2}, \ldots, \varepsilon_{n}$ are known to be i.i.d. normal with mean zero and covariance $\sigma_{\varepsilon}^{2} I_{n}$ with $\sigma_{\varepsilon}^{2}$ unknown. By using penalized spline of degree $\mathrm{q}$ for the model (1) we get:

$$
y_{i}=\sum_{j=0}^{p} \beta_{j} x_{j i}+\sum_{j=1}^{q} \beta_{p+j} x_{p+1, i}^{j}+\sum_{k=1}^{K} u_{k}\left(x_{p+1, i}-k_{k}\right)_{+}^{q}+\varepsilon_{i}, i=1,2, \ldots, n
$$

where $k_{1}, \ldots, k_{K}$ are inner knots $a<k_{1}<\ldots<k_{K}<b$. By using a convenient connection between penalized splines and mixed models, the model (2) is rewritten as follows:

$$
Y=X \beta+Z u+\varepsilon
$$

where $Y$ has length $n, X$ is a $n \times(p+q+1)$ design matrix of pure polynomial component of the spline, $Z$ is a $n \times K$ design matrix of spline truncated functions, $\beta$ is a $(p+q+1)$-vector of parameters of pure polynomial component of the spline, $u$ is a $K$-vector with spline truncated functions, and the vector of error term $\varepsilon$ has length $n, \quad \varepsilon \sim N\left(0, \sigma_{\varepsilon}^{2} I\right)$.

Assume that $u$ and $\varepsilon$ are independent and the prior distribution on $u, \pi_{0}(u)$ is $N\left(0, \sigma_{u}^{2} I\right)$, the prior distribution on the parameters vector $\beta, \pi_{0}(\beta)$ is $N\left(0, \sigma_{\beta}^{2} I\right)$, and we will assume that the prior distribution on $\sigma_{\varepsilon}^{2}, \pi_{0}\left(\sigma_{\varepsilon}^{2}\right)$ is inverse gamma $I G\left(\alpha_{\varepsilon}, \beta_{\varepsilon}\right)$, also we assume that $\sigma_{u}^{2} \sim I G\left(\alpha_{u}, \beta_{u}\right)$, where the hyperparameters $\alpha_{\varepsilon}, \beta_{\varepsilon}, \alpha_{u}, \beta_{u}$ that determine the priors and must be chosen by the statistician.

\section{Posterior Distribution}

From the model (3) we have

$$
Y \mid \theta, \sigma_{\varepsilon}^{2}, \sigma_{u}^{2} \sim N\left(C \theta, \sigma_{\varepsilon}^{2} I_{n}\right)
$$

where, $C=\left[\begin{array}{ll}X & Z\end{array}\right]$ and $\theta=\left[\begin{array}{ll}\beta & u\end{array}\right]^{T}$. Then, the likelihood function $L\left(Y \mid \theta, \sigma_{\varepsilon}^{2}, \sigma_{u}^{2}\right)$ is

$$
L\left(Y \mid \theta, \sigma_{\varepsilon}^{2}, \sigma_{u}^{2}\right) \propto\left|\sigma_{\varepsilon}^{2}\right|^{-1 / 2} \exp \left\{-\frac{1}{2}(Y-C \theta)^{T}\left(\sigma_{\varepsilon}^{2} I_{n}\right)^{-1}(Y-C \theta)\right\}
$$

Then, the posterior distributions of the vector of coefficients $\theta$ and the error variance $\sigma_{\varepsilon}^{2}$ and $\sigma_{u}^{2}$ are

$$
\begin{aligned}
& \pi_{1}\left(\theta \mid Y, \sigma_{\varepsilon}^{2}, \sigma_{u}^{2}\right) \propto L\left(Y \mid \theta, \sigma_{\varepsilon}^{2}, \sigma_{u}^{2}\right) \pi_{0}(\theta) \\
& \Rightarrow \pi_{1}\left(\theta \mid Y, \sigma_{\varepsilon}^{2}, \sigma_{u}^{2}\right) \propto \exp \left\{-\frac{1}{2}(Y-C \theta)^{T}\left(\sigma_{\varepsilon}^{2} I_{n}\right)^{-1}(Y-C \theta)\right\} \pi_{0}(\theta)
\end{aligned}
$$

and

$$
\begin{aligned}
& \pi_{1}\left(\sigma_{\varepsilon}^{2} \mid Y, \theta, \sigma_{u}^{2}\right) \propto\left|\sigma_{\varepsilon}^{2}\right|^{-1 / 2} \exp \left\{-\frac{1}{2}(Y-C \theta)^{T}\left(\sigma_{\varepsilon}^{2} I_{n}\right)^{-1}(Y-C \theta)\right\} \pi_{0}\left(\sigma_{\varepsilon}^{2}\right) \\
& \pi_{1}\left(\sigma_{u}^{2} \mid Y, \theta, \sigma_{\varepsilon}^{2}\right) \propto\left|\sigma_{\varepsilon}^{2}\right|^{-1 / 2} \exp \left\{-\frac{1}{2}(Y-C \theta)^{T}\left(\sigma_{\varepsilon}^{2} I_{n}\right)^{-1}(Y-C \theta)\right\} \pi_{0}\left(\sigma_{u}^{2}\right)
\end{aligned}
$$

From (6) we can see

$$
\theta \mid Y, \sigma_{\varepsilon}^{2}, \sigma_{u}^{2} \sim N\left(\mu_{\theta \mid Y, \sigma_{\varepsilon}^{2}, \sigma_{u}^{2}}, \sum_{\theta \mid Y, \sigma_{\varepsilon}^{2}, \sigma_{u}^{2}}\right)
$$

where

$$
\mu_{\theta \mid Y, \sigma_{\varepsilon}^{2}, \sigma_{u}^{2}}=\left\{\sum \Lambda\right\}\left\{\sigma_{\varepsilon}^{2} I_{n}+\left[\sum \Lambda C^{T} C\right]\right\}^{-1} C^{T}
$$




$$
\sum_{\theta \mid Y, \sigma_{\varepsilon}^{2}, \sigma_{u}^{2}}=\sum \Lambda-\sum^{2} \Lambda C^{T}\left\{\sigma_{\varepsilon}^{2} I_{n}+\left[\sum \Lambda C^{T} C\right]\right\}^{-1}\{C \Lambda\}
$$

and

$$
\sum=\left[\begin{array}{cc}
\sigma_{\beta}^{2} & 0 \\
0 & \sigma_{u}^{2}
\end{array}\right], \Lambda=\left[\begin{array}{cc}
I_{p+q+1} & 0 \\
0 & I_{n-(p+q+1)}
\end{array}\right]
$$

then

$$
\sum \Lambda=\left[\begin{array}{cc}
\sigma_{\beta}^{2} I_{p+q+1} & 0 \\
0 & \sigma_{u}^{2} I_{n-(p+q+1)}
\end{array}\right]
$$

Now, by spectral decomposition we obtain $C^{T} C=P D P^{T}[4]$, where $D=\operatorname{diag}\left(d_{1}, \ldots, d_{n}\right)$ is the matrix of eigenvalues and $P$ is the orthogonal matrix of eigenvectors. Thus

$$
\sigma_{\varepsilon}^{2} I_{n}+\sum \Lambda C^{T} C=\sigma_{\varepsilon}^{2} P\left\{I_{n}+\left(\begin{array}{cc}
\delta I_{p+q+1} & 0 \\
0 & \gamma I_{n-(p+q+1)}
\end{array}\right) D\right\} P^{T}
$$

where, $\delta=\frac{\sigma_{\beta}^{2}}{\sigma_{\varepsilon}^{2}}$ and $\gamma=\frac{\sigma_{u}^{2}}{\sigma_{\varepsilon}^{2}}$. Then, the conditional density of $Y$ given $\sigma_{\varepsilon}^{2}, \delta$ and $\gamma$ can be written as:

$$
\begin{gathered}
m\left(Y \mid \sigma_{\varepsilon}^{2}, \delta, \gamma\right)=\frac{1}{\left(2 \pi \sigma_{\varepsilon}^{2}\right)^{n / 2} \operatorname{det}\left[I_{n}+\left(\begin{array}{cc}
\delta I_{p+q+1} & 0 \\
0 & \gamma I_{n-(p+q+1)}
\end{array}\right) D\right]^{1 / 2}} \\
\quad \exp \left\{-\frac{1}{2 \sigma_{\varepsilon}^{2}} Y^{T} P\left[I_{n}+\left(\begin{array}{cc}
I_{p+q+1} & 0 \\
0 & \gamma I_{n-(p+q+1)}
\end{array}\right) D\right]^{-1} P^{T Y}\right\} \\
\quad \frac{1}{\left(2 \pi \sigma_{\varepsilon}^{2}\right)^{n / 2}} \frac{1}{\left[\prod_{i=1}^{p+q+1}\left[1+\delta d_{i}\right]\right]^{1 / 2}\left[\prod_{i=p+q+2}^{n}\left[1+\gamma d_{i}\right]\right]^{1 / 2}} \\
\quad \exp \left\{-\frac{1}{2 \sigma_{\varepsilon}^{2}}\left(\sum_{i=1}^{p+q+1} \frac{s_{i}^{2}}{1+\delta d_{i}}+\sum_{i=p+q+2}^{n} \frac{s_{i}^{2}}{1+\gamma d_{i}}\right)\right\}
\end{gathered}
$$

where $s=\left(s_{1}, \ldots, s_{n}\right)^{T}=P^{T} Y$.

Theorem 1. The joint posterior density of $\delta, \gamma$ given $Y$ is

$$
\begin{aligned}
& \pi_{1}(\delta, \gamma \mid Y) \propto \frac{\gamma^{(b / 2)-1} \delta^{\alpha_{\varepsilon}-1} e^{\frac{\sigma_{\beta}^{2} \delta}{\beta_{\varepsilon}}}}{(a+b \gamma)^{-(a+b) / 2}}\left(\prod_{i=1}^{p+q+1}\left(1+\delta d_{i}\right)\right)^{-1 / 2}\left(\prod_{i=p+q+2}^{n}\left(1+\gamma d_{i}\right)\right)^{-1 / 2} \\
& \left(2 \beta_{\varepsilon}+\sum_{i=1}^{p+q+1} \frac{s_{i}^{2}}{1+\delta d_{i}}+\sum_{i=p+q+2}^{n} \frac{s_{i}^{2}}{1+\gamma d_{i}}\right)
\end{aligned}
$$

Proof. Since $\delta \sim \operatorname{Gamma}\left(\alpha_{\varepsilon}, \beta_{\varepsilon}\right), \gamma \sim F(b, a)[5]$

$$
\begin{aligned}
\therefore \pi_{1}(\delta, \gamma \mid Y)= & \int m\left(Y \mid \sigma_{\varepsilon}^{2}, \delta, \gamma\right) f\left(\delta, \alpha_{\varepsilon}, \beta_{\varepsilon}\right) f(\gamma, b, a) f\left(\sigma_{\varepsilon}^{2}, \alpha_{\varepsilon}, \beta_{\varepsilon}\right) d \sigma_{\varepsilon}^{2} \\
& =\int \frac{1}{\left(2 \pi \sigma_{\varepsilon}^{2}\right)^{n / 2}}\left(\prod_{i=1}^{p+q+1}\left(1+\delta d_{i}\right)\right)^{-1 / 2}\left(\prod_{i=p+q+2}^{n}\left(1+\gamma d_{i}\right)\right)^{-1 / 2} \frac{\sigma_{\beta}^{2}}{\beta_{\varepsilon} \Gamma\left(\alpha_{\varepsilon}\right)}\left(\frac{\sigma_{\beta}^{2} \delta}{\beta_{\varepsilon}}\right)^{\alpha_{\varepsilon}-1}
\end{aligned}
$$




$$
\begin{aligned}
& \exp \left\{-\frac{1}{2 \sigma_{\varepsilon}^{2}}\left(\sum_{i=1}^{p+q+1} \frac{s_{i}^{2}}{1+\delta d_{i}}+\sum_{i=p+q+2}^{n} \frac{s_{i}^{2}}{1+\gamma d_{i}}\right)\right\} \exp \left\{\frac{\sigma_{\beta}^{2} \delta}{\beta_{\varepsilon}} \frac{b^{b / 2} a^{a / 2}}{\beta(b, a)} \frac{\gamma^{(b / 2)-1}}{(a+b \gamma)^{-(a+b) / 2}}\right\} \\
& \frac{\beta_{\varepsilon}^{\alpha_{\varepsilon}}}{\Gamma\left(\alpha_{\varepsilon}\right)}\left(\sigma_{\varepsilon}^{2}\right)^{-\left(\alpha_{\varepsilon}+1\right)} \exp \left\{-\frac{\beta_{\varepsilon}}{\sigma_{\varepsilon}^{2}}\right\} d \sigma_{\varepsilon}^{2} \\
& =(2 \pi)^{-n / 2} \frac{\left(\sigma_{\beta}^{2} \delta\right)^{\alpha_{\varepsilon}-1} e^{\frac{\sigma_{\beta}^{2} \delta}{\beta_{\varepsilon}}}}{\left(\Gamma\left(\alpha_{\varepsilon}\right)\right)^{2}} \frac{b^{b / 2} a^{a / 2}}{\beta(b, a)} \frac{\gamma^{(b / 2)-1}}{(a+b \gamma)^{-(a+b) / 2}} \\
& \int \frac{1}{\left(2 \pi \sigma_{\varepsilon}^{2}\right)^{n / 2}}\left(\prod_{i=1}^{p+q+1}\left(1+\delta d_{i}\right)\right)^{-1 / 2}\left(\prod_{i=p+q+2}^{n}\left(1+\gamma d_{i}\right)\right)^{-1 / 2} \\
& \exp \left\{-\frac{1}{2 \sigma_{\varepsilon}^{2}}\left(\beta_{\varepsilon}+\sum_{i=1}^{p+q+1} \frac{s_{i}^{2}}{1+\delta d_{i}}+\sum_{i=p+q+2}^{n} \frac{s_{i}^{2}}{1+\gamma d_{i}}\right)\right\}\left(\sigma_{\varepsilon}^{2}\right)^{-\left(n+2 \alpha_{\varepsilon}+2\right) / 2} d \sigma_{\varepsilon}^{2} \\
& =(2 \pi)^{-n / 2} \frac{\left(\sigma_{\beta}^{2} \delta\right)^{\alpha_{\varepsilon}-1} e^{\frac{\sigma_{\beta}^{2} \delta}{\beta_{\varepsilon}}}}{\left(\Gamma\left(\alpha_{\varepsilon}\right)\right)^{2}} \frac{b^{b / 2} a^{a / 2}}{\beta(b, a)} \frac{\gamma^{(b / 2)-1}}{(a+b \gamma)^{-(a+b) / 2}}(2)^{\left(n+2 \alpha_{\varepsilon}+2\right) / 2} \\
& \int \frac{1}{\left(2 \pi \sigma_{\varepsilon}^{2}\right)^{n / 2}}\left(\prod_{i=1}^{p+q+1}\left(1+\delta d_{i}\right)\right)^{-1 / 2}\left(\prod_{i=p+q+2}^{n}\left(1+\gamma d_{i}\right)\right)^{-1 / 2} \\
& \exp \left\{-\frac{1}{2 \sigma_{\varepsilon}^{2}}\left(2 \beta_{\varepsilon}+\sum_{i=1}^{p+q+1} \frac{s_{i}^{2}}{1+\delta d_{i}}+\sum_{i=p+q+2}^{n} \frac{s_{i}^{2}}{1+\gamma d_{i}}\right)\right\} \\
& \left(\frac{2 \beta_{\varepsilon}+\sum_{i=1}^{p+q+1} \frac{s_{i}^{2}}{1+\delta d_{i}}+\sum_{i=p+q+2}^{n} \frac{s_{i}^{2}}{1+\gamma d_{i}}}{2 \sigma_{\varepsilon}^{2}}\right)^{\left(n+2 \alpha_{c}\right.} \\
& \left(2 \beta_{\varepsilon}+\sum_{i=1}^{p+q+1} \frac{s_{i}^{2}}{1+\delta d_{i}}+\sum_{i=p+q+2}^{n} \frac{s_{i}^{2}}{1+\gamma d_{i}}\right)^{-\left(n+2 \alpha_{\varepsilon}+2\right) / 2} d \sigma_{\varepsilon}^{2} \\
& \propto \frac{\gamma^{(b / 2)-1} \delta^{\alpha_{\varepsilon}-1} e^{\frac{\sigma_{\beta}^{2} \delta}{\beta_{\varepsilon}}}}{(a+b \gamma)^{-(a+b) / 2}} \int \frac{1}{\left(2 \pi \sigma_{\varepsilon}^{2}\right)^{n / 2}}\left(\prod_{i=1}^{p+q+1}\left(1+\delta d_{i}\right)\right)^{-1 / 2}\left(\prod_{i=p+q+2}^{n}\left(1+\gamma d_{i}\right)\right)^{-1 / 2} \\
& \exp \left\{-\frac{1}{2 \sigma_{\varepsilon}^{2}}\left(2 \beta_{\varepsilon}+\sum_{i=1}^{p+q+1} \frac{s_{i}^{2}}{1+\delta d_{i}}+\sum_{i=p+q+2}^{n} \frac{s_{i}^{2}}{1+\gamma d_{i}}\right)\right\} \\
& \left(\frac{2 \beta_{\varepsilon}+\sum_{i=1}^{p+q+1} \frac{s_{i}^{2}}{1+\delta d_{i}}+\sum_{i=p+q+2}^{n} \frac{s_{i}^{2}}{1+\gamma d_{i}}}{2 \sigma_{\varepsilon}^{2}}\right)^{\left[\left(n+2 \alpha_{\varepsilon}+2\right) / 2\right]-1} \\
& \left(2 \beta_{\varepsilon}+\sum_{i=1}^{p+q+1} \frac{s_{i}^{2}}{1+\delta d_{i}}+\sum_{i=p+q+2}^{n} \frac{s_{i}^{2}}{1+\gamma d_{i}}\right)^{-\left(n+2 \alpha_{\varepsilon}+2\right) / 2} d \sigma_{\varepsilon}^{2} \\
& \propto \frac{\gamma^{(b / 2)-1} \delta^{\alpha_{\varepsilon}-1} e^{\frac{\sigma_{\beta}^{2} \delta}{\beta_{\varepsilon}}}}{(a+b \gamma)^{-(a+b) / 2}} \Gamma\left(\left(n+2 \alpha_{\varepsilon}+4\right) / 2\right)\left(\prod_{i=1}^{p+q+1}\left(1+\delta d_{i}\right)\right)^{-1 / 2}\left(\prod_{i=p+q+2}^{n}\left(1+\gamma d_{i}\right)\right)^{-1 / 2}
\end{aligned}
$$




$$
\begin{aligned}
& \left(2 \beta_{\varepsilon}+\sum_{i=1}^{p+q+1} \frac{s_{i}^{2}}{1+\delta d_{i}}+\sum_{i=p+q+2}^{n} \frac{s_{i}^{2}}{1+\gamma d_{i}}\right)^{-\left(n+2 \alpha_{\varepsilon}+2\right) / 2} \\
& \therefore \pi_{1}(\delta, \gamma \mid Y) \propto \frac{\gamma^{(b / 2)-1} \delta^{\alpha_{\varepsilon}-1} e^{\frac{\sigma_{\beta}^{2} \delta}{\beta_{\varepsilon}}}}{(a+b \gamma)^{-(a+b) / 2}}\left(\prod_{i=1}^{p+q+1}\left(1+\delta d_{i}\right)\right)^{-1 / 2}\left(\prod_{i=p+q+2}^{n}\left(1+\gamma d_{i}\right)\right)^{-1 / 2} \\
& \left(2 \beta_{\varepsilon}+\sum_{i=1}^{p+q+1} \frac{s_{i}^{2}}{1+\delta d_{i}}+\sum_{i=p+q+2}^{n} \frac{s_{i}^{2}}{1+\gamma d_{i}}\right)^{-\left(n+2 \alpha_{\varepsilon}+2\right) / 2}
\end{aligned}
$$

Theorem 2. The posterior mean and covariance matrix of $\theta$ are

$$
E(\theta \mid Y)=\Lambda P \quad E\left\{\left[I_{n}+\left(\begin{array}{cc}
\delta I_{p+q+1} & 0 \\
0 & \gamma I_{n-(p+q+1)}
\end{array}\right) D\right]^{-1} \mid Y\right\} C^{T} s
$$

and

$$
\begin{aligned}
& \operatorname{Var}(\theta \mid Y)=\frac{1}{n+2 \alpha_{\varepsilon}+2} E\left[\frac{\left(2 \beta_{\varepsilon}+\left(\sum_{i=1}^{p+q+1} \frac{s_{i}^{2}}{1+\delta d_{i}}+\sum_{i=p+q+2}^{n} \frac{s_{i}^{2}}{1+\gamma d_{i}}\right)\right)}{Y}\right] \Lambda- \\
& \frac{1}{n+2 \alpha_{\varepsilon}+2} \Lambda C^{T} P \quad E\left(2 \beta_{\varepsilon}+\left(\sum_{i=1}^{p+q+1} \frac{s_{i}^{2}}{1+\delta d_{i}}+\sum_{i=p+q+2}^{n} \frac{s_{i}^{2}}{1+\gamma d_{i}}\right)\right) \\
& {\left[\left[I_{n}+\left(\begin{array}{cc}
\delta I_{p+q+1} & 0 \\
0 & \gamma I_{n-(p+q+1)}
\end{array}\right) D\right]^{-1} \mid Y\right] P^{\prime} C \Lambda+} \\
& E\left\{\Lambda C^{T} P\left\{\left[I_{n}+\left(\begin{array}{cc}
\delta I_{p+q+1} & 0 \\
0 & \gamma I_{n-(p+q+1)}
\end{array}\right) D\right]^{-1}\right\} \mathrm{s}\right\}
\end{aligned}
$$

\section{Proof.}

$$
\begin{aligned}
& E(\theta \mid Y)=\mu_{\theta \mid Y}=\left\{\sum \Lambda\right\}\left\{\sigma_{\varepsilon}^{2} I_{n}+\left[\sum \Lambda C^{T} C\right]\right\}^{-1} C^{T} Y \\
& =\sum \Lambda\left\{\sigma_{\varepsilon}^{2} P\left[I_{n}+\left(\begin{array}{cc}
\delta I_{p+q+1} & 0 \\
0 & \gamma I_{n-(p+q+1)}
\end{array}\right) D\right] P^{T}\right\}^{-1} C^{T} Y \\
& =\frac{\sum}{\sigma_{\varepsilon}^{2}} \Lambda\left(P^{T}\right)^{-1}\left\{\left[I_{n}+\left(\begin{array}{cc}
\delta I_{p+q+1} & 0 \\
0 & \gamma I_{n-(p+q+1)}
\end{array}\right) D\right]\right\}^{-1} P^{-1} C^{T} Y
\end{aligned}
$$

$\because P$ is the orthogonal matrix of eigenvectors, then $P^{-1}=P^{T}$ and $\left(P^{T}\right)^{-1}=P$.

Therefore

$$
E(\theta \mid Y)=\Lambda P\left(\begin{array}{cc}
\delta I_{p+q+1} & 0 \\
0 & \gamma I_{n-(p+q+1)}
\end{array}\right)\left\{\left[I_{n}+\left(\begin{array}{cc}
\delta I_{p+q+1} & 0 \\
0 & \gamma I_{n-(p+q+1)}
\end{array}\right) D\right]\right\}^{-1} P^{-1} C^{T} Y
$$




$$
=\Lambda P \quad E\left\{\left[I_{n}+\left(\begin{array}{cc}
\delta I_{p+q+1} & 0 \\
0 & \gamma I_{n-(p+q+1)}
\end{array}\right) D\right]^{-1} \mid Y\right\} C^{T} s
$$

where the expectation $E\left\{\left[I_{n}+\left(\begin{array}{cc}\delta I_{p+q+1} & 0 \\ 0 & \gamma I_{n-(p+q+1)}\end{array}\right) D\right]^{-1} \mid Y\right\}$ taken with respect to $\pi_{1}(\delta, \gamma \mid Y)$. By following the same way we can prove the variance of $(\theta \mid Y)$ 口

\section{Model Checking and Bayes Factors}

We would like to choose between a Bayesian penalized spline semiparametric regression model as a mixed model and a Bayesian penalized spline semiparametric regression model with known coefficients by using Bayes factors for two hypotheses

$$
H_{0}: y_{i}=\sum_{j=0}^{p} \beta_{j}^{0} x_{j i}+\sum_{j=1}^{q} \beta_{j+1}^{0} x_{p+1, i}^{j}+\sum_{k=1}^{K} u_{k}^{0}\left(x_{p+1, i}-k_{k}\right)_{+}^{q}+\varepsilon
$$

versus

$$
H_{1}: y_{i}=\sum_{j=0}^{p} \beta_{j} x_{j i}+\sum_{j=1}^{q} \beta_{j+1} x_{p+1, i}^{j}+\sum_{k=1}^{K} u_{k}\left(x_{p+1, i}-k_{k}\right)_{+}^{q}+\varepsilon
$$

or

$$
\left.\begin{array}{c}
H_{0}: Y=X \beta^{0}+Z u^{0}+\varepsilon \\
\text { versus } \\
H_{1}: Y=X \beta+Z u+\varepsilon
\end{array}\right\}
$$

where $\beta^{0}$ and $u^{0}$ are known. We compute the Bayes factor, $B_{01}$, of $H_{0}$ relative to $H_{1}$ for testing problem (16) as follows

$$
B_{01}(Y)=\frac{m\left(Y \mid H_{0}\right)}{m\left(Y \mid H_{1}\right)}
$$

where $m\left(Y \mid H_{i}\right)$ is the marginal density of $Y$ under model $H_{i}, i=0,1$. We have:

$$
\begin{aligned}
m\left(Y \mid H_{0}\right)=\int f\left(Y \mid \beta^{0}, u^{0}, \sigma_{\varepsilon}^{2}\right) \pi_{0}\left(\sigma_{\varepsilon}^{2}\right) d \sigma_{\varepsilon}^{2} \\
=(2 \pi)^{-n / 2} \frac{\beta_{\varepsilon}^{\alpha_{\varepsilon}}}{\Gamma\left(\alpha_{\varepsilon}\right)} \int\left(\sigma_{\varepsilon}^{2}\right)^{-n / 2} \exp \left(\frac{\beta_{\varepsilon}}{\sigma_{\varepsilon}^{2}}\right)\left(\sigma_{\varepsilon}^{2}\right)^{-\left(\alpha_{\varepsilon}+1\right)} \exp \left(-\frac{\left(Y-X \beta^{0}-Z u^{0}\right)^{2}}{2 \sigma_{\varepsilon}^{2}}\right) d \sigma_{\varepsilon}^{2} \\
=(2 \pi)^{-n / 2} \frac{\beta_{\varepsilon}^{\alpha_{\varepsilon}}}{\Gamma\left(\alpha_{\varepsilon}\right)} \int\left(\sigma_{\varepsilon}^{2}\right)^{-\left(\frac{n}{2}+\alpha_{\varepsilon}+1\right)} \exp \left(\frac{-\beta_{\varepsilon}+\frac{1}{2}\left(Y-X \beta^{0}-Z u^{0}\right)^{2}}{2 \sigma_{\varepsilon}^{2}}\right) d \sigma_{\varepsilon}^{2} \\
\left.=(2 \pi)^{-n / 2} \frac{\beta_{\varepsilon}^{\alpha_{\varepsilon}}}{\Gamma\left(\alpha_{\varepsilon}\right)} \int\left(\sigma_{\varepsilon}^{2}\right)^{-\left(\frac{n}{2}+\alpha_{\varepsilon}+1\right)}\left(\beta_{\varepsilon}+\frac{1}{2}\left(Y-X \beta^{0}-Z u^{0}\right)^{2}\right)^{\left(\frac{n}{2}+\alpha_{\varepsilon}+1\right)}\right) d \sigma_{\varepsilon}^{2} \\
\quad\left(\beta_{\varepsilon}+\frac{1}{2}\left(Y-X \beta^{0}-Z u^{0}\right)^{2}\right)^{-\left(\frac{n}{2}+\alpha_{\varepsilon}+1\right)} \exp \left(\frac{-\beta_{\varepsilon}+\frac{1}{2}\left(Y-X \beta^{0}-Z u^{0}\right)^{2}}{2 \sigma_{\varepsilon}^{2}}\right. \\
=(2 \pi)^{-n / 2} \frac{\beta_{\varepsilon}^{\alpha_{\varepsilon}}}{\Gamma\left(\alpha_{\varepsilon}\right)} \int \frac{\left(\beta_{\varepsilon}+\frac{1}{2}\left(Y-X \beta^{0}-Z u^{0}\right)^{2}\right)^{\left(\frac{n}{2}+\alpha_{\varepsilon}+1\right)}}{\left(\sigma_{\varepsilon}^{2}\right)^{\left(\frac{n}{2}+\alpha_{\varepsilon}+1\right)}} \\
\quad \exp \left(\frac{-\beta_{\varepsilon}+\frac{1}{2}\left(Y-X \beta^{0}-Z u^{0}\right)^{2}}{2 \sigma_{\varepsilon}^{2}}\right)\left(\beta_{\varepsilon}+\frac{1}{2}\left(Y-X \beta^{0}-Z u^{0}\right)^{2}\right)^{-\left(\frac{n}{2}+\alpha_{\varepsilon}+1\right)} d \sigma_{\varepsilon}^{2}
\end{aligned}
$$




$$
\begin{aligned}
& =(2 \pi)^{-n / 2} \frac{\beta_{\varepsilon}^{\alpha_{\varepsilon}}}{\Gamma\left(\alpha_{\varepsilon}\right)} \int\left(\frac{\beta_{\varepsilon}+\frac{1}{2}\left(Y-X \beta^{0}-Z u^{0}\right)^{2}}{\sigma_{\varepsilon}^{2}}\right)^{\left(\frac{n}{2}+\alpha_{\varepsilon}+2\right)-1} \\
& \exp \left(\frac{-\beta_{\varepsilon}+\frac{1}{2}\left(Y-X \beta^{0}-Z u^{0}\right)^{2}}{2 \sigma_{\varepsilon}^{2}}\right)\left(\beta_{\varepsilon}+\frac{1}{2}\left(Y-X \beta^{0}-Z u^{0}\right)^{2}\right)^{-\left(\frac{n}{2}+\alpha_{\varepsilon}+1\right)} d \sigma_{\varepsilon}^{2} \\
& =(2 \pi)^{-n / 2} \frac{\beta_{\varepsilon}^{\alpha_{\varepsilon}}}{\Gamma\left(\alpha_{\varepsilon}\right)} \Gamma\left(\frac{n}{2}+\alpha_{\varepsilon}+1\right)\left(\beta_{\varepsilon}+\frac{1}{2}\left(Y-X \beta^{0}-Z u^{0}\right)^{2}\right)^{-\left(\frac{n}{2}+\alpha_{\varepsilon}+1\right)}
\end{aligned}
$$

and

$$
\begin{aligned}
& m\left(Y \mid H_{1}, \sigma_{\varepsilon}^{2}, \delta, \gamma\right)=\left(2 \pi \sigma_{\varepsilon}^{2}\right)^{-n / 2}\left(\prod_{i=1}^{p+q+1}\left(1+\delta d_{i}\right)\right)^{-1 / 2}\left(\prod_{i=p+q+2}^{n}\left(1+\gamma d_{i}\right)\right)^{-1 / 2} \\
& \exp \left\{-\frac{1}{2 \sigma_{\varepsilon}^{2}}\left(\sum_{i=1}^{p+q+1} \frac{s_{i}^{2}}{1+\delta d_{i}}+\sum_{i=p+q+2}^{n} \frac{s_{i}^{2}}{1+\gamma d_{i}}\right)\right\}
\end{aligned}
$$

Therefore,

$$
\begin{gathered}
m\left(Y \mid H_{1}\right)=\int m\left(Y \mid H_{1}, \sigma_{\varepsilon}^{2}, \delta, \gamma\right) \pi_{0}\left(\sigma_{\varepsilon}^{2}, \delta, \gamma\right) d \sigma_{\varepsilon}^{2} d \delta d \gamma \\
=\int \frac{\beta_{\varepsilon}^{\alpha_{\varepsilon}}}{\Gamma\left(\alpha_{\varepsilon}\right)}\left(\sigma_{\varepsilon}^{2}\right)^{-\left(\alpha_{\varepsilon}+1\right)} \exp \left(\frac{\beta_{\varepsilon}}{\sigma_{\varepsilon}^{2}}\right)\left(2 \pi \sigma_{\varepsilon}^{2}\right)^{-n / 2}\left(\prod_{i=1}^{p+q+1}\left(1+\delta d_{i}\right)\right)^{-1 / 2}\left(\prod_{i=p+q+2}^{n}\left(1+\gamma d_{i}\right)\right)^{-1 / 2} \\
\exp \left\{-\frac{1}{2 \sigma_{\varepsilon}^{2}}\left(\sum_{i=1}^{p+q+1} \frac{s_{i}^{2}}{1+\delta d_{i}}+\sum_{i=p+q+2}^{n} \frac{s_{i}^{2}}{1+\gamma d_{i}}\right)\right\} \pi_{0}(\delta, \gamma) d \sigma_{\varepsilon}^{2} d \delta d \gamma \\
=\frac{\beta_{\varepsilon}^{\alpha_{\varepsilon}}}{\Gamma\left(\alpha_{\varepsilon}\right)}(2 \pi)^{-n / 2} \int\left(\prod_{i=1}^{p+q+1}\left(1+\delta d_{i}\right)\right)^{-1 / 2}\left(\prod_{i=p+q+2}^{n}\left(1+\gamma d_{i}\right)\right)^{-1 / 2} \pi_{0}(\delta, \gamma) \\
\int\left\{\exp \left\{-\frac{1}{2 \sigma_{\varepsilon}^{2}}\left(\beta_{\varepsilon}+\sum_{i=1}^{p+q+1} \frac{s_{i}^{2}}{1+\delta d_{i}}+\sum_{i=p+q+2}^{n} \frac{s_{i}^{2}}{1+\gamma d_{i}}\right)\right\} d \sigma_{\varepsilon}^{2}\right\} d \delta d \gamma \\
\therefore m\left(Y \mid H_{1}\right)=\frac{\beta_{\varepsilon}^{\alpha_{\varepsilon}}}{\Gamma\left(\alpha_{\varepsilon}\right)}(2 \pi)^{-\mathrm{n} / 2} \Gamma\left(\frac{n}{2}+\alpha_{\varepsilon}\right) \\
\quad\left(\prod_{i=1}^{p+q+1}\left(1+\delta d_{i}\right)\right)^{-1 / 2}\left(\prod_{i=p+q+2}^{n}\left(1+\gamma d_{i}\right)\right) \pi_{0}(\delta, \gamma) \\
\quad\left(\beta_{\varepsilon}+\frac{1}{2}\left(\sum_{i=1}^{p+q+1} \frac{s_{i}^{2}}{1+\delta d_{i}}+\sum_{i=p+q+2}^{n} \frac{s_{i}^{2}}{1+\gamma d_{i}}\right)\right) d \delta d \gamma
\end{gathered}
$$

\section{$5 \quad$ Simulation Results}

In this section, we illustrate the effectiveness of our methodology, we generated observations from the model (1) with the following regression functions:

$$
\begin{aligned}
& \text { (i) } y_{1}=2+3 x_{1}+\exp \left\{\left(x_{2}+0.4\right)^{2}\right\} \\
& \text { (ii) } y_{2}=3 x_{1}+\frac{\sin \left\{12\left(x_{2}+0.2\right)\right\}}{\left(x_{2}+0.2\right)}-x_{2}^{3} .
\end{aligned}
$$

The observations for $x$ are generated from uniform distribution on the interval $[0,1]$. The sample sizes taken are $n=25,50,100,150,200$. 
The goodness of fit of the estimated models quantified by computing the criterions average mean squared error $(A M S E)$ and average mean absolute error $(A M A E)$ are defined as:

$$
\begin{aligned}
& A M S E=\frac{1}{N} \sum_{i=1}^{N} \operatorname{MSE}\left(x_{i}\right), \\
& A M A E=\frac{1}{N} \sum_{i=1}^{N} \operatorname{MAE}\left(x_{i}\right),
\end{aligned}
$$

where $M S E$ and $M A E$ are mean squared error and mean absolute error criterions respectively.

Table (1) presents summary values of the $(A M S E)$ and $(A M A E)$ for the estimation method. From this table we can see that the values of $(A M S E)$ and $(A M A E)$ when $(n=200)$ are smaller than their values for the first test function, which were (0.0005306171) and (0.000164242) respectively. While the values of $(A M S E)$ and $(A M A E)$ are smaller when $(n=200)$ for the second test function were $(0.0001630011)$ and (0.000343007) respectively. Figures (1) and (3) below show the number of iterations of Gibbs sampler used in this paper, which was (10000) iterations for the first and second test functions respectively when $(n=200)$. While figures (2) and (4) show the density estimates based on (10000) iterations of $\sigma_{\varepsilon}^{2}$ and $\sigma_{u}^{2}$ for the first and second test functions respectively when $(n=200)$.

Table 1. Results of the $(A M S E)$ and $(A M A E)$ criterions for Bayesian semiparametric regression model.

\begin{tabular}{||c|c|c|c||}
\hline Test functions & Sample size & $A M S E$ & $A M A E$ \\
\hline \hline \multirow{6}{*}{$y_{1}$} & 25 & 0.0035217013 & 0.001642312 \\
\cline { 2 - 4 } & 50 & 0.0036214631 & 0.001656452 \\
\cline { 2 - 4 } & 100 & 0.0026621641 & 0.000731483 \\
\cline { 2 - 4 } & 150 & 0.0006316071 & 0.000185535 \\
\cline { 2 - 4 } & 200 & 0.0005306171 & 0.000164242 \\
\hline \hline \multirow{6}{*}{$y_{2}$} & 25 & 0.0030001601 & 0.004203166 \\
\cline { 2 - 4 } & 50 & 0.0025130211 & 0.002063413 \\
\cline { 2 - 4 } & 100 & 0.0002110123 & 0.001022111 \\
\cline { 2 - 4 } & 150 & 0.0002001465 & 0.000406561 \\
\cline { 2 - 4 } & 200 & 0.0001630011 & 0.000343007 \\
\hline \hline
\end{tabular}

The model checking approach based on Bayes factors has been tested on simulated examples. These Bayes factors are given in table (2). From this table, it can be seen that the model corresponding to the first test function obtains the largest Bayes factor when $(n=25)$ followed by that the second test function when $(n=25)$, and the Bayes factor favors $H_{1}$ with strong evidence with all samples sizes for two test functions.

Table 2. Values of Bayes factors

\begin{tabular}{||c|c|c|c||}
\hline \hline Test functions & Sample size & $B_{01}(y)$ & Evidence \\
\hline \hline \multirow{6}{*}{$y_{1}$} & 25 & $3.116634 \times 10^{-4}$ & Strongly favors $H_{1}$ \\
\cline { 2 - 5 } & 50 & $3.028341 \times 10^{-7}$ & Strongly favors $H_{1}$ \\
\cline { 2 - 5 } & 100 & $2.765311 \times 10^{-9}$ & Strongly favors $H_{1}$ \\
\cline { 2 - 5 } & 150 & $2.067733 \times 10^{-10}$ & Strongly favors $H_{1}$ \\
\cline { 2 - 4 } & 200 & $3.773121 \times 10^{-13}$ & Strongly favors $H_{1}$ \\
\hline \hline \multirow{3}{*}{$y_{2}$} & 25 & $5.432133 \times 10^{-6}$ & Strongly favors $H_{1}$ \\
\cline { 2 - 4 } & 50 & $7.865514 \times 10^{-14}$ & Strongly favors $H_{1}$ \\
\cline { 2 - 4 } & 100 & $6.876765 \times 10^{-18}$ & Strongly favors $H_{1}$ \\
\cline { 2 - 4 } & 150 & $9.145433 \times 10^{-20}$ & Strongly favors $H_{1}$ \\
\cline { 2 - 4 } & 200 & $4.112464 \times 10^{-28}$ & Strongly favors $H_{1}$ \\
\hline
\end{tabular}



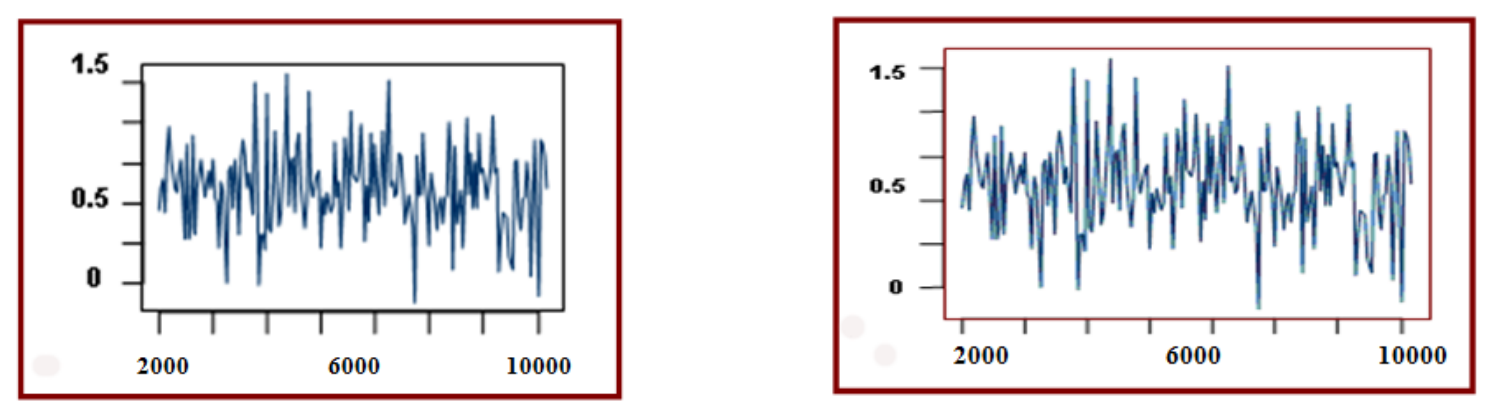

Figure 1. (10000) iterations of the Gibbs sampler for the first test function when $(n=200)$.
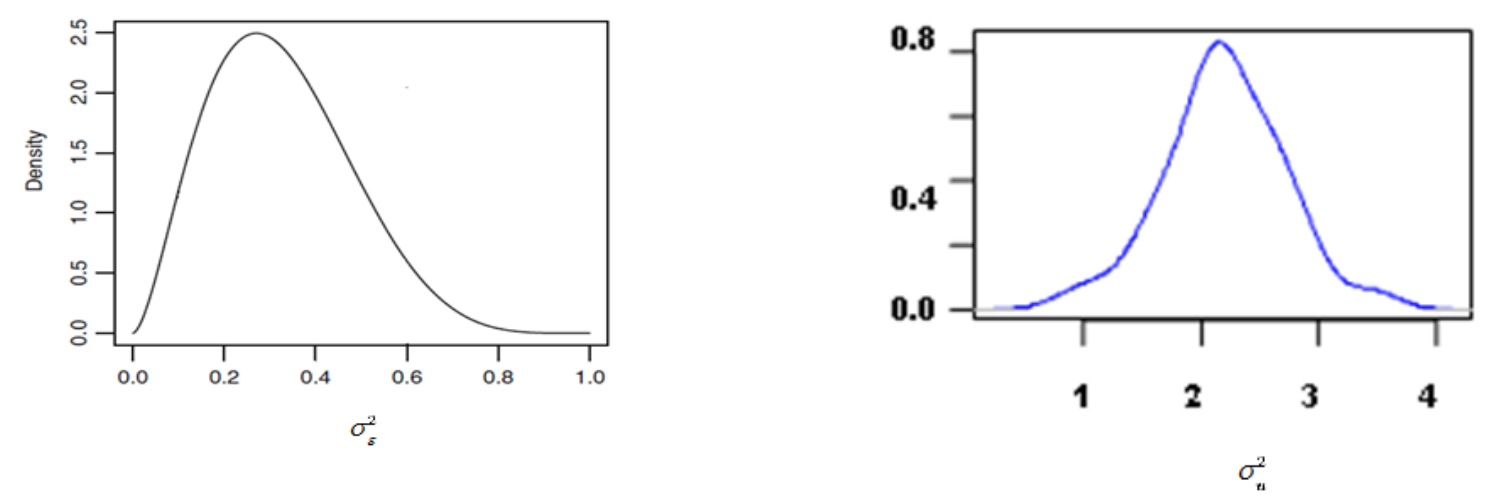

Figure 2. The density estimates based on (10000) iterations of $\sigma_{\varepsilon}^{2}$ and $\sigma_{u}^{2}$ for the first test function when $(n=200)$.
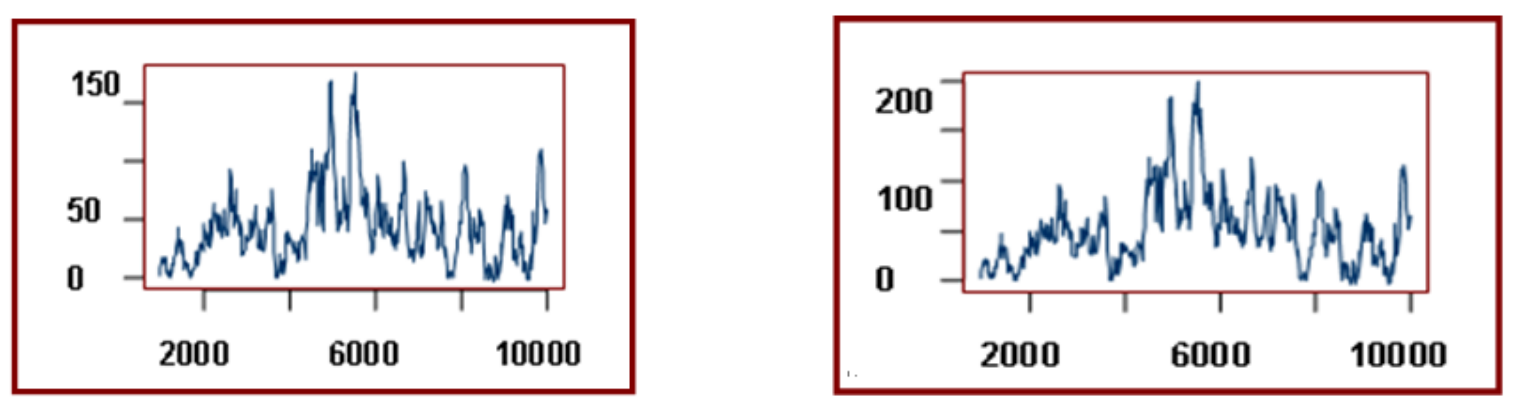

Figure 3. (10000) iterations of the Gibbs sampler for the second test function when $(n=200)$. 

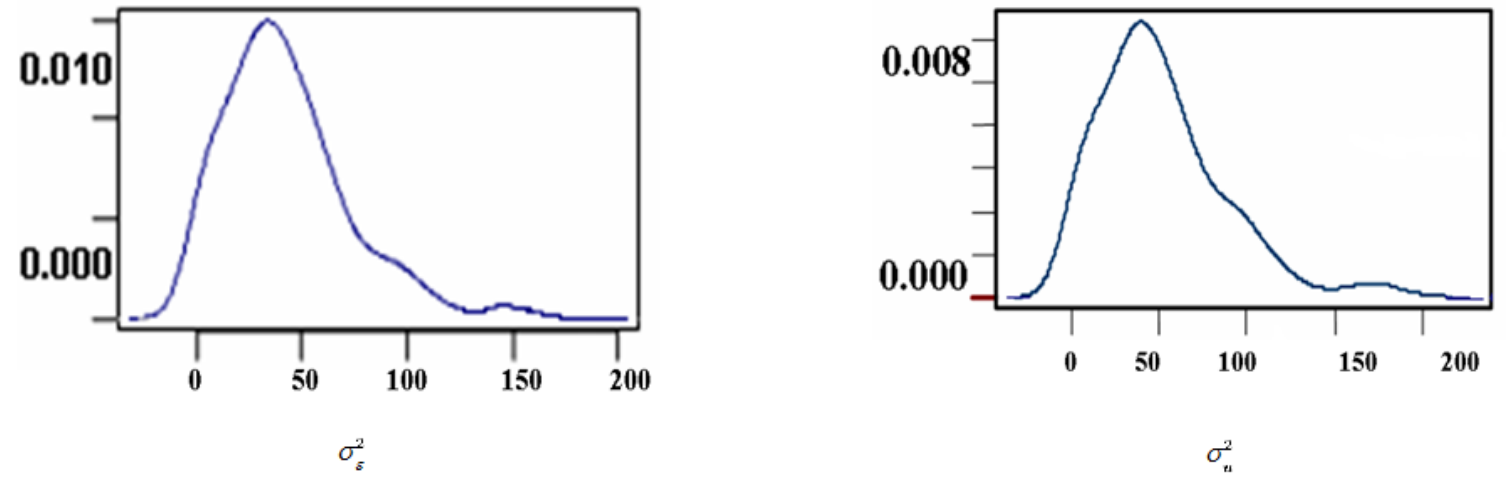

Figure 4. The density estimates based on (10000) iterations of $\sigma_{\varepsilon}^{2}$ and $\sigma_{u}^{2}$ for the second test function when $(n=200)$.

\section{Conclusions}

The conclusions obtained throughout this paper are as follows:

(1) The joint posterior density of $\delta, \gamma$ given $Y$ is

$$
\begin{gathered}
\pi_{1}(\delta, \gamma \mid Y) \propto \frac{\gamma^{(b / 2)-1} \delta^{\alpha_{\varepsilon}-1} e^{\frac{\sigma_{\beta}^{2} \delta}{\beta_{\varepsilon}}}}{(a+b \gamma)^{-(a+b) / 2}}\left(\prod_{i=1}^{p+q+1}\left(1+\delta d_{i}\right)\right)^{-1 / 2}\left(\prod_{i=p+q+2}^{n}\left(1+\gamma d_{i}\right)\right)^{-1 / 2} \\
\left(2 \beta_{\varepsilon}+\sum_{i=1}^{p+q+1} \frac{s_{i}^{2}}{1+\delta d_{i}}+\sum_{i=p+q+2}^{n} \frac{s_{i}^{2}}{1+\gamma d_{i}}\right)^{-\left(n+2 \alpha_{\varepsilon}+2\right) / 2}
\end{gathered}
$$

(2) The marginal density of $Y$ under model $H_{i}, i=0,1$ is:

$$
m\left(Y \mid H_{0}\right)=(2 \pi)^{-n / 2} \frac{\beta_{\varepsilon}^{\alpha_{\varepsilon}}}{\Gamma\left(\alpha_{\varepsilon}\right)} \Gamma\left(\frac{n}{2}+\alpha_{\varepsilon}+1\right)\left(\beta_{\varepsilon}+\frac{1}{2}\left(Y-X \beta^{0}-Z u^{0}\right)^{2}\right)^{-\left(\frac{n}{2}+\alpha_{\varepsilon}+1\right)}
$$

and

$$
\begin{gathered}
m\left(Y \mid H_{1}\right)=\frac{\beta_{\varepsilon}^{\alpha_{\varepsilon}}}{\Gamma\left(\alpha_{\varepsilon}\right)}(2 \pi)^{-n / 2} \Gamma\left(\frac{n}{2}+\alpha_{\varepsilon}\right) \int\left(\prod_{i=1}^{p+q+1}\left(1+\delta d_{i}\right)\right)^{-1 / 2}\left(\prod_{i=p+q+2}^{n}\left(1+\gamma d_{i}\right)\right)^{-1 / 2} \pi_{0}(\delta, \gamma) \\
\left(\beta_{\varepsilon}+\frac{1}{2}\left(\sum_{i=1}^{p+q+1} \frac{s_{i}^{2}}{1+\delta d_{i}}+\sum_{i=p+q+2}^{n} \frac{s_{i}^{2}}{1+\gamma d_{i}}\right)\right) d \delta d \gamma
\end{gathered}
$$

(3) In the simulation results, we concluded the following:

(a) The values of $(A M S E)$ and $(A M A E)$ when $(n=200)$ are smaller than their values for the first test function, which were (0.0005306171) and (0.000164242) respectively.

(b) The values of $(A M S E)$ and $(A M A E)$ are smaller when $(n=200)$ for the second test function were $(0.0001630011)$ and $(0.000343007)$ respectively. 
(c) The model corresponding to the first test function obtains the largest Bayes factor when $(n=25)$ followed by that the second test function when $(n=25)$.

(d) The Bayes factor favors $H_{1}$ with strong evidence with all samples sizes for two test functions.

Acknowledgments. We thank the editors and referees for providing critical comments which have brought significant improvements to our presentation.

\section{References}

1. Brezger, Andereas, Kneib, Thomas and Lang, Stefan, "Analyzing Bayesian semiparametric regression models", Work shop AG-Bayes, Universität München, 2002.

2. Choi, T., Lee, J. and Roy, A., "A note on the Bayes Factor in a Semiparametric regression model", Journal of Multivariate Analysis, 316-1327, 2008.

3. Jensen, M. J. and Maheu, J. M.,"Bayesian Semiparametric stochastic volatility modeling", The Rimini Center for Economic Analysis, Italy, 2007.

4. Jonsson, R. A. and Wichern, D. W. "Applied Multivariate Statistical Analysis" Prentice Hall, Englewood Cliffs, New Jersey 07632, 1988.

5. Jon, W., "Bayesian and frequentist regression methods, Springer New York Heidelberg Dordrecht London, (2013).

6. Lenk, P. J., "Bayesian inference for Semiparametric regression using a Fourier representation ", J.R. Statist. Soc. Ser. B61, part 4, 1999.

7. Mohaisen, A. J. and Abdulhussain, A. M., "A note on Bayes Semiparametric regression ", Mathematical Theory and Modeling www.iiste.org ISSN (Paper)2224-5804 ISSN (Online)2225-0522 Vol.3, No. 12, 2013.

8. Mohaisen, A. J. and Abdulhussain, A. M., "Bayesian Semiparametric regression with fuzzy sets ", International Journal of Pure and Applied Research in Engineering and Technology IJPRET, Vol. 2 (3):1-18 IJPRET, Research article ISSN: 2319-507X, 2013.

9. Mohaisen, A. J. and Abdulhussain, A. M., "Fuzzy sets and penalized spline in Bayesian Semiparametric regression ", LAP LAMBERT Academic Publishing, ISBN: 978-3-659-18439-0, 2014.

10. Natio, K., "Semiparametric regression with multiplicative adjustment, communications in statistics, theory and methods", 31 2289-2309, 2002.

11. Pelenis, J., "Bayesian Semiparametric Regression" Institute for Advanced Studies, Vienna 1-39, (2012).

12. Ruppert, D., Wand, M.P. and Carroll, R. J. "Semiparametric regression", Cambridge university press, (2003).

13. Temaratram, K., Robust estimation and model selection in Semiparametric regression models", Proefschrift voorgedragen tot het behalen van de grad van Doctor in de Toegepaste Economische Wetenschappen door, (2011).

14. Tsiatis, A. A. and MA, Y., "locally efficient Semiparametric estimators for functional measurement error models ", Biometrika, 91, 4, pp.835-848, 2004.

15. Wand, M.P. "Semiparametric regression and graphical models". Aust, N. Z. J. Stat., 51 (1), 2009.

16. Yuan, A. and DE Gooijer, J.G. "Semiparametric regression with kernel error model", Board of the Foundation of the Scandinavian Journal of statistics, Published by Blackwell Publishing Ltd, 2007. 